\title{
Out-of-Band Radiation from Antenna Arrays Clarified
}

\author{
Erik G. Larsson and Liesbet Van der Perre
}

\begin{abstract}
Non-linearities in radio-frequency (RF) transceiver hardware, particularly in power amplifiers, cause distortion inband and out-of-band. Contrary to claims made in recent literature, in a multiple-antenna system this distortion is correlated across the antennas in the array. A significant implication of this fact is that out-of-band emissions caused by non-linearities are beamformed, in some cases into the same direction as the useful signal.
\end{abstract}

\section{INTRODUCTION}

Non-linearities in RF amplifiers and other electronics in wireless transmitters give rise to unwanted emissions. A sinusoidal input with (angular) frequency $\omega$ that passes through a non-linearity will contain harmonic components with frequencies $\omega, 2 \omega, 3 \omega, \ldots$; see, for example, [1]. Two-tone waveforms consisting of two superimposed sinusoids with frequencies $\omega$ and $\omega^{\prime}$ will have spectral peaks at $\omega, \omega^{\prime}, \omega+\omega^{\prime}, \omega-\omega^{\prime}$, $\omega^{\prime}-\omega, 2 \omega-\omega^{\prime}, 2 \omega^{\prime}-\omega, 2 \omega+\omega^{\prime}, 2 \omega^{\prime}+\omega$, among others. Signals with a general, but band-limited, spectrum will see a widening of their bandwidth - resulting in in-band distortion inside of their nominally occupied frequency interval, as well as out-of-band distortion in adjacent frequency bands.

In-band distortion is typically quantified in terms of an error vector magnitude (EVM). This distortion translates directly into a quality loss in the demodulated signal, and this loss can be substantial. Out-of-band distortion represents, in many cases, an even more significant concern. A typical constraint is that the relative power radiated into the adjacent band shall be below a given threshold, known as the adjacent channel leakage ratio (ACLR). For example, for the LTE standard the ACLR must be below $-45 \mathrm{~dB}$. For waveforms with high peakto-average power ratios (notably, OFDM and certain multiuserMIMO precoded signals), large amplifier backoffs are required to meet specified EVM and ACLR requirements.

Recently several papers have attempted to assess the impact of hardware imperfections in transmitters equipped with multiple-antenna arrays [2], [3], especially in the context of Massive MIMO - the leading 5G physical layer technology that exploits large antenna arrays at base stations [4]. One question of concern has been whether the distortion created by hardware imperfections averages out or not, when adding more antennas - in a similar way as thermal noise averages out. Another, specific question of interest has been whether or not

E. G. Larsson is with the Dept. of Electrical Engineering (ISY), Linköping University, 58183 Linköping, Sweden. Email: erik.g.larssoneliu.se. Phone: +46-13-281312.

L. V. d. Perre is with the Dept. of Electrical Engineering, KU Leuven, 3001 Leuven, Belgium.

This work was supported in part by the Swedish Research Council (VR) and ELLIIT. the out-of-band emissions resulting from non-linearities have spatial directivity [5]-[8]. As we show in this letter, there are serious issues both with the models used, and the subsequent conclusions drawn, in several of these papers (including some by the authors).

Contribution: This letter has two specific objectives. First, we explain, from rigorous first principles, why and how the distortion resulting from non-linearities is beamformed. Second, we rectify various inaccurate statements made in recent literature.

While a mathematical analysis of non-linearities in general tends to become rather intricate, our derivations rely only on elementary manipulations of trigonometric identities. We hope that this will render the exposition accessible to a wider readership. We also discuss the implications in single-user and multiple-user communication scenarios exploiting antenna arrays.

\section{Two-Tone WAVEForm Through A THIRD-Order NON-LINEARITY}

Consider an array of antennas (indexed by $m$ ) used for beamformed transmission. The signals fed to the antennas have undergone amplification in a device with non-linear behavior generating harmonics. The question is whether the distortion caused by these non-linearities adds up constructively or not, and if so, in what spatial directions. We assume that amplification devices with identical characteristics are used on all antennas. ${ }^{1}$

To illustrate the principal phenomena, we assume that the $m$ th amplifier is fed with a two-tone signal of the form,

$$
x_{m}(t)=\cos \left(\omega_{1} t+\phi_{1}^{m}\right)+\cos \left(\omega_{2} t+\phi_{2}^{m}\right) .
$$

This is a rather simple model, but it is sufficient to prove our points (and commonly used in the analysis of non-linearities). The analysis is agnostic of $\omega_{1}$ and $\omega_{2}$, but in a practical application both would belong to a frequency interval allocated to the transmission of a useful signal (Figure 1). The signal $x_{m}(t)$ passes through a third-order memoryless non-linearity, ${ }^{2}$

$$
f(x)=x+\alpha x^{3}
$$

\footnotetext{
${ }^{1}$ Measurements have validated that small differences among the amplifiers cannot be counted on to de-correlate the distortion.

${ }^{2}$ This third-order model is relevant for many hardware components in practice. Note that second-order non-linearities are of no interest. With a second order non-linearity, $f(x)=x+\alpha x^{2}$, it can be verified that the output, after removal of frequency components far away from $\omega_{1}$ and $\omega_{2}$, and terms that constitute a scaled version of the input, is zero.
} 


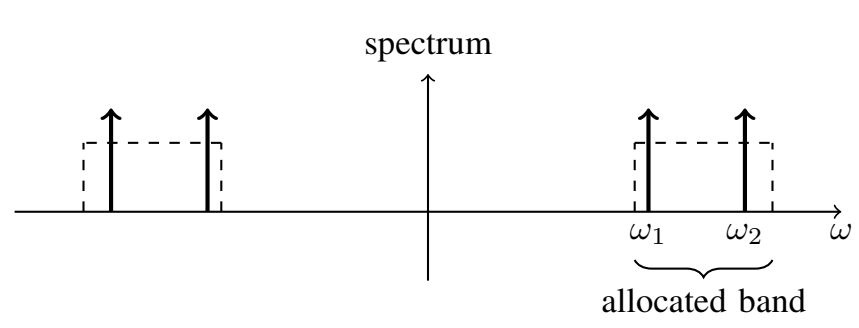

Fig. 1. Two-tone model, as representation of a signal in a frequency interval allocated to a particular transmission.

The output signal is

$$
\begin{aligned}
y_{m}(t)= & \cos \left(\omega_{1} t+\phi_{1}^{m}\right)+\cos \left(\omega_{2} t+\phi_{2}^{m}\right) \\
& +\alpha\left(\cos \left(\omega_{1} t+\phi_{1}^{m}\right)+\cos \left(\omega_{2} t+\phi_{2}^{m}\right)\right)^{3} .
\end{aligned}
$$

Through the use of standard trigonometric identities, (3) can be equivalently written as

$$
\begin{aligned}
y_{m}(t)= & \left(1+\frac{9 \alpha}{4}\right) \cos \left(\omega_{1} t+\phi_{1}^{m}\right) \\
& +\left(1+\frac{9 \alpha}{4}\right) \cos \left(\omega_{2} t+\phi_{2}^{m}\right) \\
& +\frac{3 \alpha}{4} \cos \left(2 \omega_{2} t+\omega_{1} t+2 \phi_{2}^{m}+\phi_{1}^{m}\right) \\
& +\frac{3 \alpha}{4} \cos \left(2 \omega_{2} t-\omega_{1} t+2 \phi_{2}^{m}-\phi_{1}^{m}\right) \\
& +\frac{3 \alpha}{4} \cos \left(\omega_{2} t+2 \omega_{1} t+\phi_{2}^{m}+2 \phi_{1}^{m}\right) \\
& +\frac{3 \alpha}{4} \cos \left(\omega_{2} t-2 \omega_{1} t+\phi_{2}^{m}-2 \phi_{1}^{m}\right) \\
& +\frac{\alpha}{4} \cos \left(3 \omega_{1} t+3 \phi_{1}^{m}\right) \\
& +\frac{\alpha}{4} \cos \left(3 \omega_{2} t+3 \phi_{2}^{m}\right) .
\end{aligned}
$$

The first two terms represent the desired signal, scaled by a constant. The remaining terms contain distortion created by the non-linearity. The two relevant distortion terms are those with frequencies near $\omega_{1}$ and $\omega_{2}$, that is,

$$
\begin{array}{r}
\frac{3 \alpha}{4} \cos \left(2 \omega_{2} t-\omega_{1} t+2 \phi_{2}^{m}-\phi_{1}^{m}\right) \\
\text { and } \quad \frac{3 \alpha}{4} \cos \left(\omega_{2} t-2 \omega_{1} t+\phi_{2}^{m}-2 \phi_{1}^{m}\right) .
\end{array}
$$

All other terms are irrelevant, as they have frequencies far away from $\omega_{1}$ and $\omega_{2}$ and in practice they would be suppressed by the transmit antenna frequency response and therefore effectively not transmitted.

Hence the focus now is on the terms (5) and (6), and on the question whether they add up constructively or not in particular spatial directions. To proceed with the analysis, we consider a setup with two antennas and a receiver located in the far field of the array. Then $m=1,2$.

\section{Single-user beamforming CASE}

We consider first the case of single-user beamforming. Then the two terms in (4) that constitute the desired signal are destined into the same spatial direction. Let this spatial direction be defined in terms of the difference in propagation

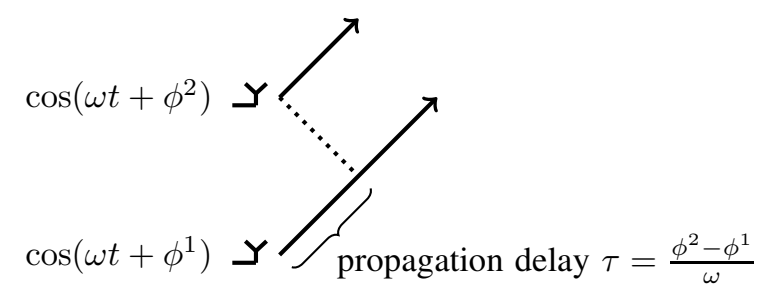

Fig. 2. Two antennas that radiate a sinusoidal signal with (angular) frequency $\omega$. If the phase difference of the sinusoids is $\phi^{2}-\phi^{1}$, then they combine constructively in the directions associated with a propagation delay of $\left(\phi^{2}-\right.$ $\left.\phi^{1}+n 2 \pi\right) / \omega$ seconds, where $n$ is an integer. If the antenna spacing is no more than half a wavelength, or equivalently the propagation delay corresponding to the antenna spacing is less than or equal to $\pi / \omega$, then there are no grating lobes and $\tau$ uniquely defines a single spatial direction.

delay, say $\tau$, between the path from the receiver to antenna 2 , respectively from the receiver to antenna 1 . Figure 2 explains the definition of $\tau$.

The two terms in (4) that represent the desired signal add up constructively in that very direction exactly if antenna 2 has a phase lead over antenna 1 of $\omega_{1} \tau$ for the first sinusoid and a phase lead of $\omega_{2} \tau$ for the second sinusoid, that is, ${ }^{3}$

$$
\begin{aligned}
& \phi_{1}^{2}-\phi_{1}^{1}=\omega_{1} \tau, \\
& \phi_{2}^{2}-\phi_{2}^{1}=\omega_{2} \tau .
\end{aligned}
$$

Suppose that the relative phases $\phi_{1}^{2}-\phi_{1}^{1}$ and $\phi_{2}^{2}-\phi_{2}^{1}$ are chosen such that (7)-(8) are satisfied. (The absolute phases $\phi_{1}^{1}$ and $\phi_{2}^{1}$ are unimportant.) Then, a direct calculation from (7)-(8) shows that,

$$
\left(2 \phi_{2}^{2}-\phi_{1}^{2}\right)-\left(2 \phi_{2}^{1}-\phi_{1}^{1}\right)=\left(2 \omega_{2}-\omega_{1}\right) \tau .
$$

Consider now the first distortion term, (5), which is a sinusoid with frequency $\left(2 \omega_{2}-\omega_{1}\right)$. For this term, antenna 2 will have a phase lead of $\left(2 \omega_{2}-\omega_{1}\right) \tau$ over antenna 1 . This means that the distortion term (5) adds up constructively in the direction defined by $\tau$ - that is, the same direction as into which the useful signal is beamformed.

A similar calculation yields the same conclusion for the second distortion term, (6). Note that with our model, if the frequencies $2 \omega_{2}-\omega_{1}$ and $\omega_{2}-2 \omega_{1}$ lie inside the band, the distortion terms impacts the EVM, otherwise they impact the ACLR.

Generally, beamforming with a linear uniform antenna array in a specific spatial direction may be achieved using either a conventional analog phased array, or a digital implementation of such an array. The spatial directivity of the out-of-band distortion ${ }^{4}$ will in the far-field have the same radiation pattern, regardless of the actual implementation of the beamforming. (See, for example, [9], for fundamental theory of radiation patterns of antenna arrays.) Considering typical radiation patterns of arrays, the presence of out-of-band distortion is not limited to one specific direction. This distortion will be present in the entire beam within which the gain is significant, as well as in the associated sidelobes. Pattern characteristics of

\footnotetext{
${ }^{3}$ All equations involving phase angles are modulo $2 \pi$.

${ }^{4}$ Except for a small extra attenuation in the antenna chain of out-of-band signals close to the in-band.
} 
harmonic and intermodulation products in broad-band active phased arrays have been investigated and reported on in the past [10], [11].

Many millimeter wave systems foreseen for $5 \mathrm{G}$ will use antenna arrays to improve the link budget, and the above analysis applies to these systems. It is likely that the power amplifiers in such systems will be operated in the non-linear region [12]. Hence, the presence of out-of-band radiation cannot be neglected.

In sub-6 GHz massive MIMO systems a similar effect will be experienced when serving one single user. Due to the randomness of path losses and shadow fading, the effective channel gain typically differs substantially between different terminals. When power control is applied to compensate for these differences and equalize the quality-of-service to the terminals, most of the radiated power is typically spent on only one of the terminals [4, p. 105]. Hence, the effect will be seen with high probability also in a multi-user scenario. However, in general, for systems operating at lower frequencies in multi-path conditions, the channel-matched beamforming will randomize the non-linear distortion terms. This fact has been established through simulations in previously published papers; see, e.g., [5].

The effect on the EVM and ACLR is as follows (for the single-user case):

- The EVM respectively ACLR values measured at the transmitter, at any of the antenna ports, are the same as in a single-antenna system. This is so because the distortion per antenna depends only on the input signal and has no dependence on intended spatial direction.

- The EVM and ACLR values measured at the receiver will also be the same as in the single-antenna case. This is so because the useful signal and the distortion receive the same array gain.

- Neither the useful signal, nor the distortion, receives any array gain in spatial directions different from that defined by $\tau$. Over-the-air measurements in other directions will show substantially smaller received distortion power than in the direction of the intended receiver.

\section{Multi-user BeAmforming CASE}

Next, consider again the two-antenna model and the case that the two sinusoids that constitute the desired signal are destined to two different spatial directions, each direction corresponding to one receiver. Let these spatial directions be defined in terms of the two delays $\tau_{1}$ respectively $\tau_{2}$, both defined analogously to $\tau$ above. The conditions for constructive combination of the desired signal are now

$$
\begin{aligned}
& \phi_{1}^{2}-\phi_{1}^{1}=\omega_{1} \tau_{1}, \\
& \phi_{2}^{2}-\phi_{2}^{1}=\omega_{2} \tau_{2} .
\end{aligned}
$$

In this case the distortion terms (5) respectively (6) add up coherently in the directions defined by

$$
\begin{aligned}
\tilde{\tau}_{1} & =\frac{\left(2 \phi_{2}^{2}-\phi_{1}^{2}\right)-\left(2 \phi_{2}^{1}-\phi_{1}^{1}\right)}{2 \omega_{2}-\omega_{1}} \\
\text { and } \quad \tilde{\tau}_{2} & =\frac{\left(\phi_{2}^{2}-2 \phi_{1}^{2}\right)-\left(\phi_{2}^{1}-2 \phi_{1}^{1}\right)}{\omega_{2}-2 \omega_{1}} .
\end{aligned}
$$

In general, it holds that

$$
\begin{array}{ll}
\tilde{\tau}_{1} \neq \tau_{1}, & \tilde{\tau}_{1} \neq \tau_{2} \\
\tilde{\tau}_{2} \neq \tau_{1}, & \tilde{\tau}_{2} \neq \tau_{2} .
\end{array}
$$

Consequently, the distortion does add up constructively in distinct spatial directions, but these directions are different from those of the two intended receivers.

Here the (transmitter) EVM and ACLR measured per antenna port are the same as in the single-antenna case, and the same as in Section III. In contrast, the EVM and ACLR measured at the receiver are better. This is so because the useful signal receives the maximum possible array gain $(3 \mathrm{~dB}$ with two antennas), whereas the distortion does not. There will, however, be other directions into which the distortion receives array gain and over-the-air measurements in those directions will show larger received distortion power.

\section{Discussion of Recent Literature}

Much recent literature has been concerned with modeling the effects of the distortion that arises from hardware impairments such as non-linearities, especially in the Massive MIMO context. For example, [2] argues that "the aggregate residual transceiver impairments in the hardware" can be modeled by additive distortion noise terms that are statistically independent among the antennas. The same model is subsequently used in, for example, [3], [13], [14]. If the distortion noise terms were statistically independent among the antennas, the distortion would effectively be radiated omni-directionally from the array. To understand why, consider a fictitious receiver located in the electrical far-field of the array. This receiver would see a sum of statistically independent distortion noise terms that add up over the air. Only the sum of the power of these noises matters, and this sum power only depends on the distance to the array - but not on the array geometry, nor on the relative array-to-receiver geometry. But, as demonstrated in Sections III-IV, the hardware non-linearities lead to spatially directive emissions of distortion. Hence, in conclusion, the distortion terms are correlated between the antennas, which means that the model of [2] is physically inaccurate.

Importantly, whether or not any "appropriate compensation algorithms have been applied" such that the focus can be on "residual" hardware impairments (an assumption made in [2]) appears to be irrelevant. If such compensation results in the complete removal of the distortion, the entire issue is immaterial. Conversely, if not, then the analysis in Sections IIIIV applies; effectively, a model such as (2) then captures the characteristics of the "compensated" hardware components.

As suggested in [8], the final result in terms of EVM within the band may in certain cases be the same when comparing a physically correct behavioral model of the non-linearities, and the independent-noise model of [2]. Yet, in many cases - and in particular, if applied to the analysis of single-user beamforming, the assumption of independent distortion terms leads to incorrect conclusions.

We next discuss out-of-band emissions more specifically. This topic has been the subject of many recent academic papers, but unfortunately, the conclusions in several of these 
papers are unclear or incorrect. This observation is important, given the current debate on out-of-band emissions in 5G standardization and regulatory discussions [15]. Space limitations permit only short comments:

- The paper [5] declares that (under assumptions specified therein) "... the absolute amount of disturbing power a victim that operates in an adjacent band suffers from is also reduced in the massive MIMO system, even if the ACLR in the single-antenna system and the MIMOACLR in the massive MIMO system are the same." But as shown above, in the single-user case, the out-of-band radiation is beamformed into the same direction as the useful signal, hence receiving the same array gain. This means that "the absolute amount of disturbing power" is not necessarily reduced.

- More seriously, the paper [7] states that "We show analytically that [out-of-band] OOB does not recombine and the array gain is experienced only inside the desired bandwidth." But as the above analysis demonstrates, constructive recombination of the distortion can occur regardless of $\omega_{1}$ and $\omega_{2}$. In particular, out-of-band radiation may recombine constructively even in the same direction as does the useful signal (Section III). Hence, the cited claim from [7] is correct only under very specific conditions, which do not hold in general.

- The paper [6] claims in its abstract that "the impact of non-linear PAs and the resulting linear and non-linear multi-user interference, quantified in terms of the received signal-to-interference-plus-noise ratio (SINR), is largely dependent on the effective or observable linear gain in the user equipment (UE) receiver demodulation stage." But this statement is inaccurate. The observable gain of the desired signal at the receiver is essentially the array gain; however, the amount of received out-of-band radiation is not a direct function of this gain. In fact, as illustrated in Section IV, in a multi-user scenario, the out-of-band radiation generally is beamformed into other directions than is the useful signal. In this case, the array gains of the useful signal and that of the distortion are different; so are the observable linear gains of these signals.

\section{CONCLUSION}

In a multiple-antenna transmitter, the distortion that results from hardware non-linearities is correlated between the antennas. This stands in contradiction to hardware impairment models popularized in the communication theory literature [2], and which postulate that distortion is statistically independent among the antennas.

This observation has several implications:

- In the single-user beamforming case, the distortion resulting from non-linearities is beamformed into the same direction as the desired signal.

- In the multi-user beamforming case, the distortion resulting from non-linearities is beamformed into distinct spatial directions, which are different from those of the desired signals.

Out-of-band interference may be the most serious form of distortion. Given that this interference is beamformed into specific directions, per-antenna ACLR constraints might be over-conservative in many cases. An alternative may be to stipulate ACLR constraints on the signal measured over-theair at the intended receiver location. However, such a constraint would not guarantee with certainty that no other locations observe strong out-of-band interference.

When finalizing this letter it was brought to our attention that some of these conclusions have already been experimentally validated: [16] demonstrated that out-of-band relative power measurements taken at different points in space were different from measurements taken at the antenna ports.

\section{ACKNOWLEDGMENT}

The authors would like to thank collaborators in the FP7MAMMOET project, and co-authors of [2], [5]-[8], for useful discussions on hardware impairments.

\section{REFERENCES}

[1] F. Horlin and A. Bourdoux, Digital Compensation for Analog FrontEnds: A New Approach to Wireless Transceiver Design. Wiley, 2008.

[2] E. Björnson, J. Hoydis, M. Kountouris, and M. Debbah, "Massive MIMO systems with non-ideal hardware: Energy efficiency, estimation, and capacity limits," IEEE Trans. Inf. Theory, vol. 60, no. 11, pp. 7112 7139, 2014.

[3] X. Zhang, M. Matthaiou, M. Coldrey, and E. Björnson, "Impact of residual transmit RF impairments on training-based MIMO systems," IEEE Trans. Commun., vol. 63, no. 8, pp. 2899-2911, 2015.

[4] T. L. Marzetta, E. G. Larsson, H. Yang, and H. Q. Ngo, Fundamentals of Massive MIMO. Cambridge University Press, 2016.

[5] C. Mollén, U. Gustavsson, T. Eriksson, and E. G. Larsson, "Out-of-band radiation measure for MIMO arrays with beamformed transmission," in Proc. IEEE Int. Conf. on Commun. (ICC), 2016.

[6] Y. Zou, O. Raeesi, L. Antilla, A. Hakkarainen, J. Vieira, F. Tufvesson, Q. Cui, and M. Valkama, "Impact of power amplifier nonlinearities in multi-user massive MIMO downlink," in IEEE Globecom Workshops, 2015.

[7] S. Blandino, C. Desset, A. Bourdoux, L. Van der Perre, and S. Pollin, "Analysis of out-of-band interference from saturated power amplifiers in massive MIMO," in European Conference on Networks and Communications (EuCNC), 2017.

[8] U. Gustavsson, C. Sanchéz-Perez, T. Eriksson, F. Athley, G. Durisi, P. Landin, K. Hausmair, C. Fager, and L. Svensson, "On the impact of hardware impairments on massive MIMO," in IEEE Globecom Workshops, 2014, pp. 294-300.

[9] C. A. Balanis, Antenna Theory: Analysis and Design. WileyInterscience, 2005.

[10] W. Sandrin, "Spatial distribution of intermodulation products in active phased array antennas," IEEE Trans. Antennas and Propagation, vol. 21, no. 6, pp. 864-868, Nov 1973.

[11] C. Hemmi, "Pattern characteristics of harmonic and intermodulation products in broadband active transmit arrays," IEEE Trans. Antennas and Propagation, vol. 50, no. 6, pp. 858-865, Jun 2002.

[12] Y. Zou, P. Zetterberg, U. Gustavsson, T. Svensson, A. Zaidi, T. Kadur, W. Rave, and G. Fettweis, "Impact of major RF impairments on mmwave communications using OFDM waveforms," in IEEE Globecom Workshops, 2016.

[13] A. K. Papazafeiropoulos and T. Ratnarajah, "Downlink MIMO HCNs with residual transceiver hardware impairments," IEEE Commun. Lett., vol. 20 , no. 10, pp. 2023-2026, 2016.

[14] J. Zhu, D. W. K. Ng, N. Wang, R. Schober, and V. K. Bhargava, "Analysis and design of secure massive MIMO systems in the presence of hardware impairments," IEEE Trans. Commun., vol. 16, no. 3, pp. 2001-2016, 2017.

[15] FCC discussion document. [Online]. Available: https://apps.fcc.gov/edocs_public/attachmatch/FCC-15-138A1.pdf

[16] E. Sienkiewicz, N. McGowan, B. Göransson, T. Chapman, and T. Elfström, "Spatially dependent ACLR modelling," in IEEE Conference on Antenna Measurements \& Applications (CAMA), 2014. 